\title{
Liver Fibrosarcoma
}

National Cancer Institute

\section{Source}

National Cancer Institute. Liver Fibrosarcoma. NCI Thesaurus. Code C5832.

A usually aggressive malignant neoplasm arising from the liver. It is characterized by the presence of spindle-shaped fibroblasts and collagenous stroma formation in a herring bone growth pattern. 